\title{
Abstract neuronal choice signals during embodied decisions
}

\author{
Florian Sandhaeger*1,2,3,4 Nina Omejc ${ }^{1,2,3,4}$, Anna-Antonia Pape ${ }^{1,2,3,4}$, Markus Siegel*1,2,3 \\ ${ }^{1}$ Hertie Institute for Clinical Brain Research, University of Tübingen, Germany \\ ${ }^{2}$ Centre for Integrative Neuroscience, University of Tübingen, Germany \\ ${ }^{3}$ MEG Center, University of Tübingen, Germany \\ ${ }^{4}$ Graduate Training Centre of Neuroscience, International Max Planck Research School, University of Tübingen, Germany \\ *Correspondence to: florian.sandhaeger@uni-tuebingen.de, markus.siegel@uni-tuebingen.de
}

\section{Abstract}

Humans can make abstract choices independent of motor actions. However, little is known about the functional role and neural representation of abstract choices. Here, we show that in the human brain choices are represented in an abstract, motor-independent manner, even when they are directly linked to an action. To disentangle sensory, choice, and motor aspects of decision-making, we measured MEG signals while human participants made choices with known and unknown motor response mapping. Using multivariate decoding, we found stimulus, choice and response information with distinct cortical distributions. Choice representations were invariant to whether or not the response mapping was known during stimulus presentation. Furthermore, neuronal choice representations predicted decision confidence and occupied distinct representational spaces from both stimulus and motor signals. Our results uncover abstract neuronal choice signals that generalize to embodied decisions. This suggests a general role of an abstract stage in human decision-making. 


\section{Introduction}

Sensory decisions are often linked to an appropriate motor action. This has led to a framework of choices emerging as action intentions ${ }^{1}$, which has been supported by numerous studies showing action-specific choice signals in motor- and premotor areas of the brain ${ }^{2-5}$. In addition, humans and other primates also make abstract choices when a suitable action is not known in advance $^{6}$. Behavioral tasks with a variable mapping of choices to motor responses have identified neuronal representations of abstract choices ${ }^{7-14}$. However, it is unclear whether abstract choice representations generalize to choices that are linked to actions.

Empirical results comparing choice signals in action-linked and action-independent situations are sparse, and do not provide a coherent picture. While some recent work found perceptual choice representations to depend on the ability to plan motor actions ${ }^{5,10}$ or response modality ${ }^{15-17}$, other previous evidence suggests at least partially overlapping representations of perceptual choices with specified or unspecified motor actions ${ }^{7}$. Furthermore, it is not known how abstract choice representations evolve across the brain because neuronal recordings were so far limited to only a small number of brain regions in isolation ${ }^{18}$.

To address this, we investigated human brain activity underlying flexible sensorimotor choices using magnetoencephalography (MEG). The task design and a multivariate analysis framework allowed us to pinpoint abstract neural choice signals independently of both sensory input and motor behavior in an action-linked as well as in an action-independent context. Abstract choice representations were present in both contexts and indistinguishable between them, suggesting a general role for abstraction in human decision-making. 


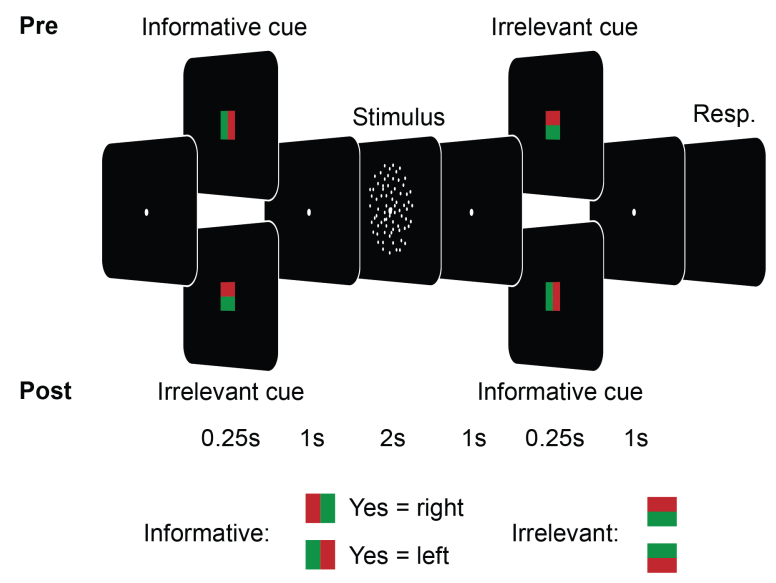

Fig. 1 | Flexible sensorimotor decision-making task. Participants reported the presence of coherent downwards motion ("yes" or "no") with a right- or left-hand button press. Mapping between choice and response was instructed by an informative cue either before (pre-condition, cue 1) or after the stimulus (post-condition, cue 2). Additionally, there was an irrelevant cue offering no additional information either after (pre-condition, cue 2) or before (post-condition, cue 1).

\section{Results}

\section{Behavior in a flexible sensorimotor decision-making task}

We recorded MEG in 33 human participants, while they performed variants of a sensorimotor decision-making task (Fig. 1). Participants decided whether a dynamic random dot stimulus contained coherent downwards motion or not. To separate stimulus-related neuronal signals from choice-related signals, we adapted the stimuli for each participant such that they performed near threshold. To disentangle choice- and motor response-related signals, we introduced a flexible mapping between choices and left- or righthand button presses that was cued on a trial-by-trial basis. For half of the trials, the choice-response mapping was revealed before stimulus onset ('pre-condition'), such that emerging choices could immediately be linked to the appropriate motor response. For the other half ('post-condition'), we revealed the mapping after stimulus offset, such that participants had to make abstract choices initially, before later selecting their motor response. Participants performed equally well on "pre" and 
"post" trials ( $74 \%$ and $73 \%$ correct; $t_{25}=1.55, P=0.13$, two-tailed t-test), and neither choice was preferentially associated with a particular motor response $(50 \%$ "right" responses for both "yes" and "no" choices, $t_{25}=0.58, P=0.57$ and $t_{25}=-0.09, P=0.93$, two-tailed t-test).

\section{Decoding neuronal representations of stimulus, response and choice-response mapping}

For each task condition separately, we quantified neuronal information about the stimulus, response, choice-response mapping and choice using a multivariate analysis approach (crossvalidated MANOVA ${ }^{19,20}$ ). Importantly, this analysis isolated neural information for each individual variable, independently of the others. Thus, for example, choice information was the information contained in the neural data about a participant's perceptual choice independent of all other variables. We found significant information about all task variables in both conditions $(P<0.01$, cluster-permutation statistics, Fig. 2). Stimulus information rose after stimulus onset and remained partially present after stimulus offset. Response information built up after stimulus offset; it did so earlier in the pre-condition where the choice-response mapping was already known during stimulus presentation. Choice-response mapping information peaked upon presentation of the relevant cue; after the pre-cue in the pre-condition and after the postcue in the post-condition. 


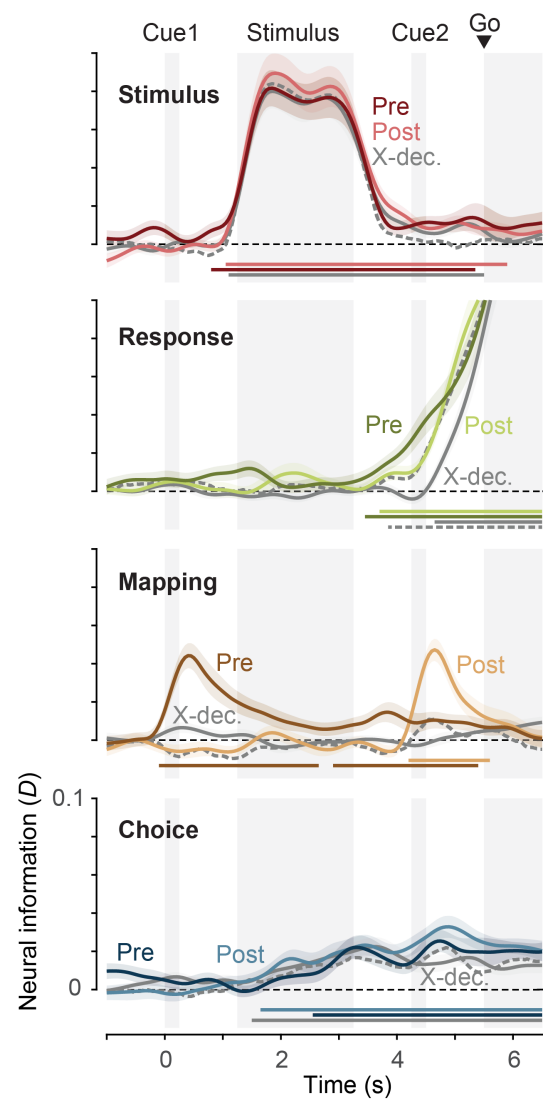

Fig. 2 | Neural information about the stimulus, response, mapping and choice. Darker lines indicate information during the post-condition, brighter lines during the pre-condition. Gray lines show the cross-decoding ('X-dec.') between both conditions, dashed gray lines the cross-decoding expected if representations in both contexts were identical. Horizontal lines denote temporal clusters of significant information (colored lines, $P<$ 0.01 , cluster-permutation, one-tailed, $\mathrm{N}=26$ ), cross-information (gray, two-tailed) or significantly less crossinformation than expected (dashed gray, one-tailed). Shaded regions indicate SEM.

\section{Abstract choice representations generalize between task contexts}

Crucially, we also found information about the perceptual choice (Fig. 2, bottom, $P<0.0001$ in both 'pre' and 'post' conditions, cluster permutation). Even though participants' choices were related to the presented stimuli and behavioral responses, our analysis framework ensured that choice information could not be explained by neural variability due to either stimuli or responses. Thus, choice information was stimulus- and response-independent.

While, in the 'post'-condition, the required motor action was not specified until after the stimulus, choices could be immediately mapped to the appropriate response in the 'pre'- 
condition. Nevertheless, choice information was present in both conditions with a similar magnitude and time course $(P>0.05$ for all time points, two-tailed t-test), rising during stimulus presentation and remaining present until the end of the trial. Choice information could not be explained by eye movements (Supplementary Fig. 1). Thus, choices were represented abstractly in the human brain, regardless of whether they could be directly linked to an action or not.

We employed a cross-decoding approach to assess the extent to which these choice representations were similar between both task conditions. We trained a decoding model on one task condition and tested it on the other. If the multivariate neural patterns distinguishing choices were identical in the 'pre'- and 'post'-conditions, we would expect the magnitude of the resulting cross-information to be comparable to the information found within the individual conditions. If, on the other hand, choices were represented in orthogonal neural populations in both conditions, cross-information should be much lower or negligible.

Cross-decoding of choices was positive throughout the trial $(P=0.0001$, cluster permutation). Furthermore, the magnitude of cross-information was similar to the magnitude of choice information in the 'pre'- and 'post'-conditions. To quantify this, we derived an estimate of the expected cross-information under the assumption of identical representations in both conditions. We found that cross-decoded choice information was never significantly lower than expected if representations were identical $(P>0.05$ for all time points). Thus, abstract choice representations were not only present but were also shared between an actionlinked and an action-independent choice context. 


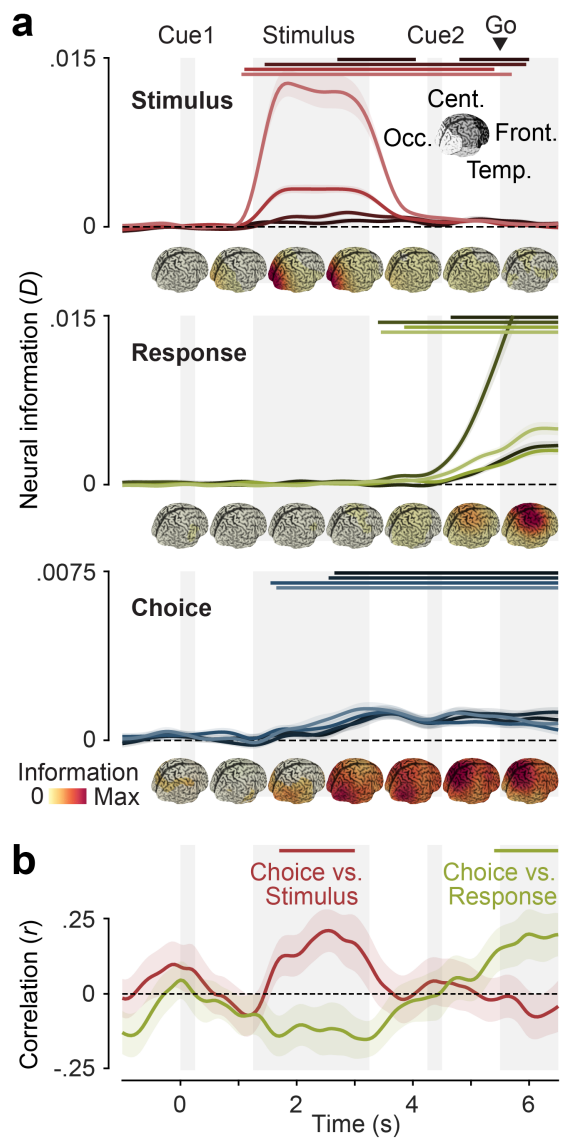

Fig. 3 Spatiotemporal dynamics of neural information. a, Time-resolved stimulus (top), response (middle) and choice (bottom) information in four groups of sources (in descending order of brightness: occipital, temporal, central, frontal). Data from both hemispheres was averaged. The cortical distribution of information during different time intervals is shown underneath the time-courses. $\mathbf{b}$, Correlation of the cortical distribution of choice information with the distribution of peak stimulus information (red) and peak response information (yellow). Horizontal lines denote temporal clusters of significant information (a, $P<0.05$, cluster-permutation, one-tailed, $\mathrm{N}=26$ ) or correlation ( $\mathrm{Bb} P<0.05$, cluster-permutation, one-tailed, $\mathrm{N}=26$ ).

\section{Choice representations dynamically shift from sensory to motor areas}

We further investigated the properties of neuronal stimulus, choice and response representations by pooling data from both task conditions. We repeated the decoding analysis in a searchlight fashion across the cortex to extract the spatiotemporal evolution of neuronal information about each variable (Fig. 3). During stimulus presentation, stimulus information was strongest in occipital visual cortex, in line with early visual representations of the sensory input. After stimulus offset, information remained at a lower level, uniformly across the brain 
(Fig. 3a, top). Response information increased earliest and most strongly in motor areas (Fig. 3a, middle), consistent with preparatory activity related to the upcoming motor response.

The expected cortical distribution and temporal evolution of choice information is less clear. Choices may be represented in visual areas, consistent with findings of choice probabilities in sensory neurons reflecting either the effect of sensory noise on decision formation or high-level feedback onto sensory populations ${ }^{18,21,22}$. Choice-specific signals may also be present in motor- and premotor areas, supporting the planning of potential motor responses $^{7,17,18,23}$, or in associative areas specialized for decision formation.

We found that the distribution of choice information changed dynamically over the course of the trial, rising first in occipital areas, before spreading throughout the brain. After the go cue, choice information remained strongest in parietal cortex and central motor areas (Fig. 3a, bottom). Given the apparent shift of choice information from occipital areas during stimulus presentation to central areas during the response phase, we quantified the similarity the cortical distribution of choice information exhibited with those of stimulus and response information. We found a significant correlation between the cortical distributions of choice and stimulus information during stimulus presentation, and between choice and response information during the response phase (Fig. 3b, stimulus: $P=0.027$, response: $P=0.045$, cluster permutation). We found similar results when repeating the searchlight analysis independently for pre- and post-condition trials (Supplementary Fig. 2). 
a
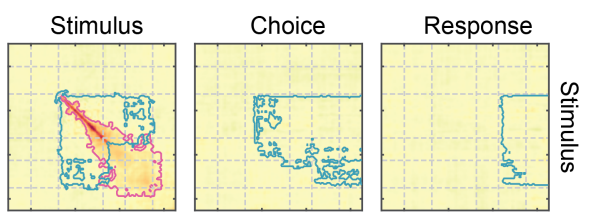

b Orthogona
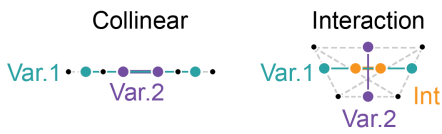

C Choice - Stim.

d Choice - Resp.

e 'yes' / 'no'
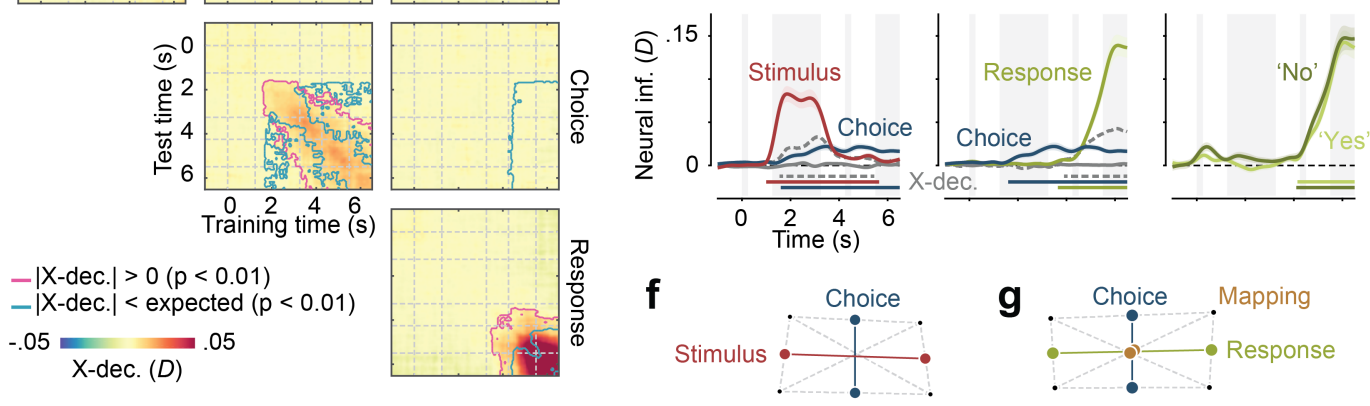

$-\mid X-$ dec. $\mid>0(p<0.01)$

$-\mid X-$ dec. $\mid<\operatorname{expected}(p<0.01)$

X-dec. (D)

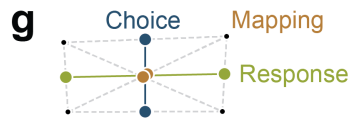

Fig. 4 | Relationship between stimulus, choice, and response representations. a, Cross-temporal and crossvariable decoding. Colors indicate neuronal information when trained and tested on any pair of time-points and variables. Pink outlines indicate clusters of shared information between timepoints and variables $(\mid \mathrm{X}-\mathrm{dec}$. $\mid>0$, cluster-permutation, $P<0.01, \mathrm{~N}=26$, two-tailed), blue outlines different representations between timepoints and variables $(\mid \mathrm{X}$-dec. $\mid<$ expected, one-tailed). b, Possible relationships between the representations of two variables. Points indicate average activity patterns for different conditions, distances between points the strength of information. Representations may be orthogonal, collinear, or orthogonal but linked with an interaction. $\mathbf{c}$ and $\mathbf{d}$, Cross-variable decoding between choice and stimulus, and choice and response, respectively. Colored lines show neural information about each variable, gray lines cross-variable information (X-dec.), and dashed gray lines the expected cross-information if both variables were represented identically. Horizontal lines indicate clusters of significant information (colored, $P<0.01$, one-tailed), or significantly less cross-information than expected (dashed gray, $P<0.01$, one-tailed). e, Response information for "yes" and "no" choices. f, Visualization of the relationship between stimulus and choice representations, based on the cross-decoding values in (c). Stimulus and choice are nearly orthogonal. g, Visualization of the relationship between choice and response representations, including mapping as their interaction, based on (d) and (e). Choice and response representations are nearly orthogonal, and response representations are equally strong for both choices. Thus, there is no systematic relation between the patterns encoding choice, stimulus and response.

\section{Temporal stability of neuronal representations}

The spatial overlap between choice, stimulus and response information raised the question whether there were shared representations between stimulus and choice during evidence accumulation and between choice and response during motor execution, respectively. We used cross-temporal and cross-variable decoding to test this, and evaluated both the temporal 
dynamics of representations and the relationships between stimulus, choice and response representations (Fig. 4a).

First, we focused on the temporal dynamics of representations. Cross-temporal decoding can reveal both time periods of relative stability ${ }^{24}$, and, when compared to the expected cross-temporal decoding between identical representations, periods of relative dynamics ${ }^{25}$. Stimulus information was initially highly dynamic, but stable after stimulus offset (Fig. 4a, top left). Given our use of fixed random dot patterns, this was consistent with stimulus information being driven by two components: During stimulus presentation, information was likely dominated by moment-to-moment differences in retinal input. After stimulus offset, the global motion content may have contributed more strongly. Choice information was temporally more stable; still, early and late choice representations were distinct (Fig. 4a, center), in line with the spatial shift from sensory to motor areas.

\section{Choice representations are distinct from sensory and motor representations}

How did the neuronal representations of different variables relate to each other? The multivariate patterns that encode any two variables are either orthogonal, indicating nonoverlapping underlying populations, collinear, indicating indistinguishable circuits underlying both representations, or somewhere in between (Fig. 4b). Furthermore, the representation of one variable may differ depending on the value of the other, i.e. the two variables may interact. In the present data, stimulus- and choice representations may depend on identical underlying circuits. For example, sensory neurons may show the same responses for visually presented as for imagined motion ${ }^{13,26}$. If such neurons strongly contributed to stimulus- and choice representations, we would expect positive cross-information between stimulus and choice. In contrast, if choice and stimulus information were driven by distinct populations, this may result in no cross-information; Our results were compatible with the latter scenario. There was no 
significant cross-decoding between stimulus and choice (Fig. 4a, top center, biggest cluster: $P$ $=0.2214$; Fig. $4 \mathrm{c}, P>0.05$ for all time points), and cross-decoding was significantly lower than expected for identical representations $(P<0.0001)$.

Next, we investigated the relationship between choice- and response representations. Again, we found no cross-information between the two variables, indicating that neural choice and response representations did not overlap (Fig. $4 \mathrm{~d}, P=0.40$ ). After selection of a motor response, choices may still have been represented as a modulation of the motor signal, e.g. leading to a relative strengthening of the activity pattern associated with the upcoming motor response for "yes"-choices compared to "no"-choices. We thus assessed the magnitude of response information, separately for each choice. However, we found no difference between both conditions ( $P=0.35$, cluster permutation, biggest cluster), indicating that even during response execution, choices were not represented as a modulation of neuronal motor activity. (Fig. 4e). We further visualized these results geometrically, which well illustrated the orthogonality of choice- and stimulus-, or choice- and response signals, respectively (Fig. 4fg). In sum, the neuronal circuit patterns underlying choice information in our MEG data were not shared with those underlying stimulus and response information, even when they were strongest in similar areas.

Abstract choice signals may also be related to, and caused by, sequential choice biases, i.e. preceding choices ${ }^{18,27,28}$. We therefore repeated the analysis including the previous choice as an additional variable. We found that choice information could not be explained by the previous choice (Supplementary Fig. 3). While our analysis already excluded the possibility that choice information was driven by overall differences between stimuli, it could theoretically still be explained by a difference between correct and error trials for one of the two stimuli. To eliminate this possibility, we trained the choice decoding model on all trials and evaluated it separately on correct and incorrect trials. This revealed no difference between both cases. Thus, 
choice information could not be explained by choice accuracy (Supplementary Fig. 4). Abstract choice information was therefore independent of previous choices and accuracy as potentially confounding variables.

\section{The strength of choice signals predicts decision confidence}

These results suggested that choice signals reflected an abstract decision stage which was neither directly related to stimulus nor to motor representations. According to signal detection theory, a hallmark of an internal decision variable is its relation to decision confidence ${ }^{29-32}$. Such a decision variable would track the available evidence supporting either choice, while confidence would be computed from the absolute distance to the decision boundary.

To enable us to assess whether the choice signals found here could reflect an internal decision variable, participants reported their choices with one of two buttons per choice, thereby additionally indicating their confidence. We repeated our decoding analysis, now adding decision confidence as an additional variable, and hypothesized that, if choice information reflected an internal decision variable, it would be stronger when confidence was high. Indeed, we found significantly stronger choice representations in trials with high confidence than in those with low confidence (Fig. 5a, $P=0.042$, cluster permutation, onetailed). We additionally performed this analysis separately for the pre-cue and post-cue task conditions, after excluding the factor of response from our model in order to retain a sufficient number of trials per condition. There was a similar pattern in both tasks (Supplementary Fig. 5, pre: $P=0.013$, post: $P=0.02$, cluster permutation, one-tailed). This indicates that the strength of choice representations predicted choice confidence, independently of whether an action could already be planned or not. Again, we visualized these results geometrically, which showed an interaction between choice and confidence representations (Fig. 5b). In contrast, 
there was no such relationship between choice confidence and the strength of motor representations (Fig. 5c, $P=0.40$, cluster permutation).
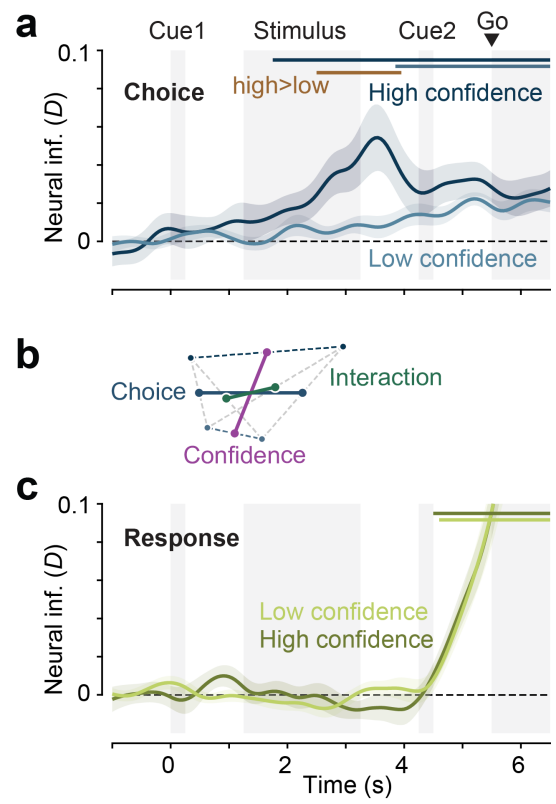

Fig. 5 | The strength of choice representations predicts confidence. a, Time-resolved choice decoding in high (dark blue) and low (light blue) confidence trials. b, Geometric visualization of choice and confidence representations and their interaction. Choice information is higher in confident trials, leading to an interaction between both variables. $\mathbf{c}$, Time-resolved response decoding in high (dark green) and low (light green) confidence trials. Horizontal lines denote temporal clusters of significant information (colored, $P<0.01$, cluster-permutation, one-tailed, $\mathrm{N}=19)$ or differences in information magnitude (gold) $(P=0.042$, cluster-permutation, one-tailed, $\mathrm{N}$ $=19)$.

\section{Discussion}

Studies of the neural basis of sensorimotor decision-making have often neglected abstract, motor-independent choices ${ }^{33}$. This is rooted in the fact that many real-world choices appear to be choices between motor actions ${ }^{1}$ and in the difficulty of accessing signals representing purely abstract choices. On the one hand, in animal studies, which provide the majority of evidence in support of neural circuits selective for specific choice options, behavioral tasks that 
disentangle choices from motor responses are very challenging. Non-invasive human studies, on the other hand, struggle to read out choice contents, and thus mostly provide indirect evidence for choice-related neural activity. Consequently, studies comparing the representation of choices in abstract and action-linked contexts are rare. A small number of notable exceptions have provided inconclusive results ${ }^{5,7,10}$, thus leaving the role and extent of abstract choice signals elusive.

By combining non-invasive MEG in humans with an advanced multivariate analysis framework, we robustly read out abstract choice contents from whole-brain neural activity. In accordance with current theories ${ }^{31,32}$, abstract choice representations depended on decision confidence, appearing to reflect the underlying decision variable. In sum, our findings point to an important role of abstraction in decision-making, even in a simple task involving a known sensorimotor mapping.

Abstract choices were not only represented in brain activity when decisions had to be made abstractly, but also when the sensorimotor mapping was known in advance. Importantly, choice representations in both task contexts were indistinguishable from one another. While the fundamental limits of MEG spatial resolution and sensitivity prevent the conclusion that the underlying circuit representations are identical, this striking similarity requires any remaining differences between conditions to be small and of a type that MEG is blind to.

Our finding of abstract choice representations generalizing between contexts in which actions can be planned and those in which they cannot is in line with behavioral evidence suggesting analogous mechanisms underlying decision-making in action-linked and independent contexts ${ }^{34}$. Furthermore, recordings in macaque area LIP have found the choice selectivity of neurons to be similar, regardless of whether a motor action was specified ${ }^{7}$. Our results extend this finding to the whole-brain level, indicating that the dominant sources of choice-selective signals generalize between contexts. This also accords well with research 
implicating a centro-parietal positivity (CPP) as an electrophysiological marker of evidence accumulation $^{35,36}$. The CPP exhibits several properties of a domain-general, abstract neural decision variable; however, while it gradually builds up with the absolute amount of evidence, it has not been shown to carry information about the choice itself. The CPP, as an unsigned marker of integration, and the specific choice signals found in the present study may reflect different aspects of the same underlying process.

The cortical distribution of abstract choice signals may be modulated by response modality. Recent work using fMRI suggested that, for vibrotactile comparisons, abstract choice representations are present in non-overlapping, modality-specific cortical areas ${ }^{15,16}$. On the other hand, direct neural recordings have shown the representation of recognition and categorization choices in medial frontal cortex to generalize between manual and saccadic responses $^{12}$. The accessibility of such modality-independent representations of choice likely depends on the specific behavioral task and type of neural measurement. Research combining multiple measurement scales ${ }^{37-39}$ should help resolving this. Our results only have indirect implications for the modality-dependence of choice signals because participants eventually always reported their choice using a button press. Nonetheless, the broad availability of choice representations across the brain, in conjunction with the shift of the information peak from visual sensory to motor areas is consistent with the co-existence of modality-independent and modality-specific components.

Perceptual decisions involve a complex interaction of feedforward and feedback processes throughout the brain ${ }^{18,40}$. Here, we found that the spatial peak of abstract choice information shifted throughout the trial, reflecting the currently relevant stage ${ }^{18,41}$. This does not necessitate that choices originate in sensory cortex and are later relayed to motor cortex; in fact, choices may be computed elsewhere, but be preferentially accessible in currently engaged areas. The global availability of choice information is in line with either a distributed 
computation, or a global broadcast of choice signals ${ }^{18,22}$. Further studies including invasive and manipulative approaches are required to pinpoint where and by which mechanisms abstract choices are computed.

A growing body of evidence has related the formation of action-linked sensorimotor decisions to activity in motor- and premotor areas ${ }^{2,3,5,23,33,40}$. Our findings are well compatible with these results: the presence of an abstract choice stage does not preclude the simultaneous planning and competition of multiple response options ${ }^{23,42}$. Indeed, using part of the dataset described here, we previously found fluctuations in motor cortical activity to predict upcoming motor responses, independently of the perceptual choice ${ }^{43,44}$. Taken together, these findings support a multi-level model of decision-making involving simultaneous evaluation of abstract choices as well as motor actions ${ }^{45}$. The relevance of an abstract choice level may be understood in light of phenomena like perceptual priors ${ }^{46,47}$, sequential choice biases ${ }^{28,43}$, or value computations associated with the choices themselves ${ }^{48}$, which all require and act on abstract choice representations. The primate brain, which is able to assess abstract options and treat decision-making problems as arbitrary categorization ${ }^{11,49}$, may do so even when not strictly necessary. Importantly, this can still be reconciled with an intentional framework of decisionmaking, if intentions are not only about actions, but also rules, or activations of neural circuits in general $^{31,45}$.

We conclude that an abstract choice stage may be universally present in human perceptual decision-making, enabling the evaluation of motor-independent choice options even during embodied decisions. 


\section{Online Methods}

\section{MEG recordings}

\section{Participants}

33 healthy, right-handed human volunteers (18 female; mean age: 28 years; 3 years SD) participated in this study and received monetary reward. All participants had normal or corrected-to-normal vision and gave written informed consent before participating. The study was conducted in accordance with the Declaration of Helsinki and approved by the ethics committee of the University of Tübingen.

\section{Behavioural task \& Stimuli}

Participants performed a flexible sensorimotor decision-making task. In each trial, they had to decide whether a random dot kinematogram contained coherent downwards motion or not and reported their choice with a left- or righthand button press. Crucially, the mapping between response hand and choice varied on a trial by trial basis. Moreover, the mapping was either revealed before (pre-condition) or after (post-condition) the stimulus. Additionally, an irrelevant cue was presented after (pre-condition) or before (post-condition) the stimulus.

Participants started a trial by acquiring fixation on a fixation spot. After a fixation period, the first cue appeared for $250 \mathrm{~ms}$, followed by a delay of $1000 \mathrm{~ms}$, the presentation of the random dot stimulus for $2000 \mathrm{~ms}$, another $1000 \mathrm{~ms}$ delay and the second cue for $250 \mathrm{~ms}$. A third $1000 \mathrm{~ms}$ delay was followed by a $33 \mathrm{~ms}$ dimming of the fixation spot, which served as the go-cue for the participant's response. The response consisted in a button press using the left or right index finger, according to the choice and the choice-response mapping. Participants chose one of two buttons on either side to indicate whether they were confident in their choice or not. $250 \mathrm{~ms}$ after their response, participants received a $100 \mathrm{~ms}$ visual feedback (centrally presented circle, 2.1 degree diameter, red for incorrect or green for correct). 
The random dot stimuli consisted of 1500 white dots with a diameter of 0.12 degrees, presented in an 8.5 degree diameter circular aperture on a black background. Dots moved at a speed of 10 degrees per second. For each participant, we used only two stimuli: First, a target stimulus, in which, on each frame, a fraction of dots moved coherently downwards, whereas the rest moved in random directions. Secondly, a noise stimulus, in which all dots moved in random directions. The motion coherence of the target stimuli was titrated to each participant's individual perceptual threshold using a staircase procedure. Choice-response cues and irrelevant cues all had the same luminance and size (0.85 degree diameter).

Each participant took part in two recording runs of one of two task versions which differed in the details of the choice-response cue as well as the confidence report. Participants 1-20 performed version A: here, the choice-response cue consisted of a centrally presented red or green square (yes=right hand: green; yes=left hand: red), whereas the irrelevant cue was a blue square. The outer button always indicated a confident, the inner one an unconfident choice. In this version, the fixation baseline at the beginning of each trial lasted $1500 \mathrm{~ms}$. Each recording run consisted of 400 randomly ordered trials, of which 120 were pre-cue trials, 120 post-cue trials, and 160 belonged to one of two control conditions not reported here. Participants 21-33 performed version B: here, the choice-response cue consisted of two vertical rectangles (yes=right hand: left rectangle mint, right rectangle pink; yes=left hand: left rectangle pink, right rectangle mint) forming a square, whereas the irrelevant cue consisted of two horizontal rectangles (upper: pink, lower: mint). The confidence mapping (inner or outer button for confident / unconfident responses) was changed in each recording run. Here, the fixation baseline was $1000 \mathrm{~ms}$. Each run consisted of 400 randomly ordered trials, 200 of which were pre-cue and 200 post-cue trials.

To ensure that participants were performing both task conditions well, we computed overall accuracy as the percentage of correct trials. We used a two-tailed paired t-test to test 
whether accuracy was different between task conditions. To make sure participants did not systematically associate one of the motor responses with one of the choices, we computed the percentage of "right" button presses for "yes"- and "no" choices separately and compared both against $50 \%$ using two-tailed t-tests.

\section{Setup \& recording}

We recorded MEG (Omega 2000, CTF Systems, Inc., Port Coquitlam, Canada) with 275 channels at a sampling rate of $2,343.75 \mathrm{~Hz}$ in a magnetically shielded chamber. Participants sat upright in a dark room, while stimuli were projected onto a screen at a viewing distance of $55 \mathrm{~cm}$ using an LCD projector (Sanyo PLC-XP41, Moriguchi, Japan) at $60 \mathrm{~Hz}$ refresh rate. Stimuli were constructed offline and presented using the Presentation software (NeuroBehavioral Systems, Albany, CA, USA). To ensure continuous fixation, we recorded eye movements using an Eyelink 1000 system (SR Research, Ottawa, Ontario, Canada).

\section{MEG preprocessing and source analysis}

\section{Preprocessing}

We low-pass-filtered MEG and eye-tracking data at $10 \mathrm{~Hz}$ (two-pass forward-reverse Butterworth filter, order 4) and down-sampled to $20 \mathrm{~Hz}$. Trials containing eye-blinks were rejected. We chose not to apply a high-pass filter in order to avoid filter artefacts ${ }^{50}$. At the same time, we could not use a baseline correction as choice effects could plausibly be driven by previous trials. We thus used robust detrending ${ }^{51}$ to remove polynomial trends from the MEG data, but not the eye tracking data, in a piecewise fashion (600 s pieces, removal of linear trend followed by $10^{\text {th }}$ order polynomial). Data of three participants was rejected due to metal artifacts. 


\section{Source reconstruction}

For source reconstruction based on each participant's individual anatomy, we recorded structural T1-weighted MRIs (echo time $(\mathrm{TE})=2.18 \mathrm{~ms}$, repetition time $(\mathrm{TR})=2.3 \mathrm{~ms}$, longitudinal relaxation time $(\mathrm{T} 1)=1.1 \mathrm{~ms}$, flip angle $=9^{\circ}, 192$ slices, voxel size $\left.1 \times 1 \times 1 \mathrm{~mm}^{3}\right)$ with a Siemens 3T Tim Trio scanner and a 32 channel Head Coil. We generated single-shell head models ${ }^{52}$ and estimated three-dimensional (x, y and z-direction) MEG source activity at 457 equally spaced locations $7 \mathrm{~mm}$ beneath the skull, using linear spatial filtering ${ }^{53}$. We retained, for each source, activity in all three directions and concatenated the data of the two separate recording runs per participant. For all subsequent analyses, we used subsets of this 1371-dimensional source space: for most whole-head decoding analyses we selected 229 approximately equally distributed sources, for each of which we summed the three dipole directions in order to decrease the dimensionality. The only exception was the whole-head decoding analysis including the previous choice (Supplementary Fig. 3); due to the lower number of trials that could be included in this analysis and the resulting lower number of degrees of freedom, we only used 115 sources. Due to the inherently low spatial resolution of MEG, which leads to a much lower effective dimensionality than the number of measurement channels ${ }^{54}$, these sub-selections were unlikely to significantly affect the results. For searchlight analyses, we used each of the 457 sources' immediate neighbors, including all 3 dipole directions.

\section{Multivariate decoding analyses}

\section{Task variables and cross-validation scheme}

The experimental design resulted in a number of variables of which each trial instantiated a combination. For each trial, we defined the task (pre- or post-cue), stimulus (target or noise), response (left or right hand button-press), mapping (target $=$ left or target $=$ right), choice 
(yes/target or no/noise), accuracy (correct or incorrect) and confidence (high or low). Not all of these variables were independent of each other: for a given stimulus and choice, accuracy is fixed; and for a given choice and mapping, response is fixed. Thus, 5 independent variables giving rise to 32 conditions remained. While those variables under experimental control (task, stimulus, mapping) were fully balanced, those dependent on the participants' behavior (choice, response, confidence, accuracy) were not, leading to a non-uniform sampling of conditions. To ensure an accurate estimation of neural information about each variable, independent of the others, we implemented an n-fold cross-validation scheme, where n was the lowest trial count per condition. Thus, for each cross-validation fold, both training and test data contained trials of all conditions. In order to decrease the dependence of our results on a particular random partition into folds, we repeated each analysis 10 times, with different random seeds. All results were averaged across these random seeds before further processing.

Due to the variability in behavioral responses, as well as the rejection of trials containing eyeblink artefacts, we did not retain sufficient trials from each condition for all participants. For our main analyses (Fig. 2, Fig. 3, Fig. 4, Supplementary Fig. 1, Supplementary Fig. 2, Supplementary Fig. 4), including task, stimulus, choice, response, mapping and accuracy as variables, data from 26 participants had sufficient trials. When additionally including confidence as a variable, but neglecting the task condition (Fig. 5), we retained 19 participants. To assess the effect of confidence separately for both task conditions (Supplementary Fig. 5), we used all variables apart from response, leading again to 19 useable participants. To assess the effect of the previous choice in relation to the current choice (Supplementary Fig. 3), we neglected the task condition as well as confidence, and included stimulus, choice, response, mapping, accuracy, and previous choice. This left us with data from 22 participants. For all decoding analyses, we combined source level data from both recording runs per participant. 
To do so, we normalized the data per channel, time point and run over trials, and then concatenated data of both runs.

\section{Cross-validated MANOVA}

We used cross-validated MANOVA (cvMANOVA; 11, 12) to estimate the amount of information in multivariate MEG data about the task variables of interest. Here, we implemented cvMANOVA for time-resolved data, including the capability of cross-decoding by training and testing the model on different time points, variables, or levels of any variable. To this end, we first estimated a baseline noise covariance matrix, using trials from all unique conditions. We then "trained" the model by estimating contrasts of beta weights of each unique condition in a cross-validation fold's training set, and "tested" it by estimating contrasts of beta weights in the fold's test set. An estimate of true pattern distinctness was computed as the dot product of these contrasts, normalized by the noise covariance:

$$
D=\operatorname{trace}\left(\frac{1}{n} B_{\text {train }}^{\prime} C_{\text {train }} C_{\text {train }}^{-1} X_{\text {test }}^{\prime} X_{\text {test }} C_{\text {train }} C_{\text {test }}^{-1} B_{\text {test }} \Sigma^{-1}\right)
$$

where $X_{\text {test }}$ is the design matrix indicating the unique condition of each trial in the test set, $C_{\text {train }}$ is the contrast vector the model is trained on, $C_{\text {test }}$ the test contrast vector and $\Sigma^{-1}$ the inverted noise covariance matrix. $B_{\text {train }}$ and $B_{\text {test }}$ contained the regression parameters of a multivariate general linear model

$$
\begin{gathered}
B_{\text {train }}=X_{\text {train }}^{-1} Y_{\text {train }} \\
B_{\text {test }}=X_{\text {test }}^{-1} Y_{\text {test }}
\end{gathered}
$$


where $Y_{\text {train }}$ and $Y_{\text {test }}$ are the training and test data sets. The inverted noise covariance matrix $\Sigma^{-1}$ was estimated using data from a baseline timepoint $(-0.5 \mathrm{~s}$ with respect to the onset of the first cue):

$$
\begin{gathered}
B_{\text {train }_{b l}}=X_{\text {train }_{\text {train }_{b l}}^{-1}{ }} \\
\Xi=Y_{\text {train }_{b l}}-X_{\text {train } B_{\text {train }_{b l}}} \\
\Sigma^{-1}=(f E-p-1) \cdot\left(\Xi^{\prime} \Xi\right)^{-1}
\end{gathered}
$$

with $f E$ being the degrees of freedom and $p$ the number of sources used. $\Xi$ was regularized towards the unity matrix using a regularization parameter of 0.05 .

While cvMANOVA technically constitutes an encoding framework - modelling data variability due to experimental variables - it shares many similarities with commonly used multivariate decoding methods ${ }^{55}$. Notably, cvMANOVA uses out-of-sample cross-validation to provide a measure of the information contained in neural data about the variables of interest. These estimates can, in principle, also be used to decode experimental variables on individual trials. Due to this close relationship, and to highlight the link to the extensive multivariate decoding literature, we often refer to our results as decoding results.

\section{Cross-decoding}

To achieve cross-condition decoding, we constructed contrast vectors $C_{\text {train }}$ and $C_{\text {test }}$ to only contain the conditions to be trained or tested on, respectively. We applied this to estimate neural information within and across the two task conditions (pre and post), as well as the two confidence levels, and the two choices. Additionally, we also used a model trained on all trials, 
and tested it separately on correct and incorrect trials. To estimate whether information was shared between timepoints, we computed the pattern distinctness when using regression parameters $B_{\text {train }}$ from one time point, and $B_{\text {test }}$ from another. We repeated this for every pair of time points. In order to assess whether two variables shared a common representational space, we used cross-variable decoding. We implemented this by using a training contrast $C_{\text {train }}$ differentiating between the levels of one variable, and a test contrast $C_{\text {test }}$ differentiating between the levels of another. Before further processing, all decoding timecourses were smoothed using a Hanning window (500 ms, full width at half maximum). Time-time generalization matrices were smoothed using a 2-dimensional, $100 \mathrm{~ms}$ Hanning window.

\section{Geometric visualization of representational similarity}

We reconstructed low-dimensional geometric representations of neural activity in multiple conditions using the decoding results. Decoding- and cross-decoding values between multiple variables define the distances and angles of condition difference vectors. We used these to plot subsets of conditions in 2D-spaces.

\section{Searchlight analysis}

We repeated our main analysis in a searchlight fashion, in order to estimate the spatiotemporal distribution of neural information throughout the trial. For each of the 457 sources, we used cvMANOVA on that source as well as its immediate neighbors, including all 3 dipole directions. In order to maintain comparability between sources, we normalized the resulting pattern distinctness values by the square root of the size of the searchlight ${ }^{19,20}$. After averaging over both hemispheres, we split the searchlight decoding results of all 457 sources into four distinct groups (occipital, temporal, central, frontal) and averaged within each of these areas, to show the spatiotemporal dynamics of neural information. To quantify a shift in choice 
information from sensory to motor areas, we correlated, for each participant, the cortical distribution of choice information during each timepoint with the distribution of stimulus information during stimulus presentation $(1.25 \mathrm{~s}$ to $3.25 \mathrm{~s})$, and with the distribution of response information during response execution (from $5.5 \mathrm{~s}$ ). Statistical significance was assessed using one-tailed cluster-permutation tests.

\section{Expected cross-decoding}

The maximal amount of shared information between two contexts depends on the amount of information available in each individual context. Thus, in order to assess whether two representations are different, the strength of both representations has to be taken into account and compared with the strength of the shared representation. We thus estimated the expected cross-decoding

$$
E_{12}=\sqrt{\left|D_{1} D_{2}\right|} \cdot \operatorname{sign}\left(D_{1}\right) \cdot \operatorname{sign}\left(D_{2}\right)
$$

where $D_{1}$ and $D_{2}$ denote the pattern distinctness in the two contexts. The cross-decoding $D_{12}$ between both contexts would be expected to approach $E_{12}$ for identical representations. Any cross-decoding values smaller than $\mathrm{E}_{12}$ indicate that the representations are not fully overlapping.

\section{Eye movement control}

While we ensured continuous fixation using an online eye movement control at the beginning of each trial, small eye movements can still plausibly confound MEG signals ${ }^{56}$. We thus repeated our main decoding analysis (Fig. 2) using eye-tracking data. For this purpose, we selected the x-position, y-position, and pupil size signals and averaged them over both eyes. 
Additionally, we computed the eye position eccentricity as $\operatorname{sqr}\left(\mathrm{x}^{2}+\mathrm{y}^{2}\right)$. We then applied the same decoding analysis using cvMANOVA, using these 4 channels. We split the 26 participants into the 13 with the highest and lowest choice information in their eye signals, respectively. This revealed that in a subset of participants eye signals were predictive of choice. To test whether this could plausibly explain the neural choice information, we compared the choice decoding timecourses in both splits. As neural choice decoding was, if anything, weaker in those participants with higher choice decoding from the eye signals, the neural decoding was unlikely to be explained by eye movements (Supplementary Fig. 1).

\section{Statistical analysis}

We assessed the statistical significance of information using cluster-based sign permutation tests. After determining temporally contiguous clusters during which pattern distinctness was higher than 0 (one-tailed t-test over participants, $P<0.05$ ), we randomly multiplied the information time-course of each participant 10,000 times with either 1 or -1 . In each random permutation, we re-computed information clusters and determined the cluster-mass of the strongest cluster. Each original cluster was assigned a p-value by comparing its size to the distribution of sizes of the random permutation's strongest clusters. The same procedure was used for cross-decoding analyses, however using two-tailed t-tests as true cross-decoding can also be negative. We also tested differences in information using this strategy, namely between response information during "yes" and "no"-choices (Fig. 4e), between choice or response information in high and low confidence trials (Fig. 5a, b; Supplementary Fig. 5), and between choice information in correct and incorrect trials (Supplementary Fig. 4). To determine whether two representations were significantly different, we tested whether the empirical crossdecoding was smaller than the expected cross-decoding, again using cluster-based sign 
permutation tests. Cross-temporal generalization and dynamics were assessed analogously, however using two-dimensional clusters.

\section{Software}

All analyses were performed in MATLAB, using custom code as well as the Fieldtrip 57 and SPM toolboxes.

\section{Data and materials availability}

Preprocessed MEG data and analysis code to reproduce all reported results is available from the authors on reasonable request. 


\section{References}

1. Cisek, P. \& Kalaska, J. F. Neural Mechanisms for Interacting with a World Full of Action Choices. Annual Review of Neuroscience 33, 269-298 (2010).

2. Shadlen, M. N. \& Newsome, W. T. Neural Basis of a Perceptual Decision in the Parietal Cortex (Area LIP) of the Rhesus Monkey. Journal of Neurophysiology 86, 1916-1936 (2001).

3. Gold, J. I. \& Shadlen, M. N. Representation of a perceptual decision in developing oculomotor commands. Nature 404, 390-394 (2000).

4. Gold, J. I. \& Shadlen, M. N. The Neural Basis of Decision Making. Annual Review of Neuroscience 30, 535-574 (2007).

5. Wang, M. et al. Macaque dorsal premotor cortex exhibits decision-related activity only when specific stimulus-response associations are known. Nature Communications 10, (2019).

6. Gold, J. I. \& Shadlen, M. N. The Influence of Behavioral Context on the Representation of a Perceptual Decision in Developing Oculomotor Commands. The Journal of Neuroscience 23, 632-651 (2003).

7. Bennur, S. \& Gold, J. I. Distinct Representations of a Perceptual Decision and the Associated Oculomotor Plan in the Monkey Lateral Intraparietal Area. Journal of Neuroscience 31, 913-921 (2011).

8. Hebart, M. N., Donner, T. H. \& Haynes, J.-D. Human visual and parietal cortex encode visual choices independent of motor plans. NeuroImage 63, 1393-1403 (2012).

9. Horwitz, G. D., Batista, A. P. \& Newsome, W. T. Representation of an Abstract Perceptual Decision in Macaque Superior Colliculus. Journal of Neurophysiology 91, 2281-2296 (2004). 
10. Ludwig, S., Herding, J. \& Blankenburg, F. Oscillatory EEG signatures of postponed somatosensory decisions. Human Brain Mapping 39, 3611-3624 (2018).

11. Merten, K. \& Nieder, A. Active encoding of decisions about stimulus absence in primate prefrontal cortex neurons. Proceedings of the National Academy of Sciences 109, 62896294 (2012).

12. Minxha, J., Adolphs, R., Fusi, S., Mamelak, A. N. \& Rutishauser, U. Flexible recruitment of memory-based choice representations by the human medial frontal cortex. Science 368, eaba3313 (2020).

13. Zhou, Y. \& Freedman, D. J. Posterior parietal cortex plays a causal role in perceptual and categorical decisions. Science 6 (2019).

14. Merten, K. \& Nieder, A. Comparison of abstract decision encoding in the monkey prefrontal cortex, the presupplementary, and cingulate motor areas. Journal of Neurophysiology 110, 19-32 (2013).

15. Wu, Y., Velenosi, L. A., Schröder, P., Ludwig, S. \& Blankenburg, F. Decoding vibrotactile choice independent of stimulus order and saccade selection during sequential comparisons. Human Brain Mapping 40, 1898-1907 (2019).

16. Wu, Y., Velenosi, L. A. \& Blankenburg, F. Response modality-dependent abstract choice representations for vibrotactile comparisons. bioRxiv (2019) doi:10.1101/802652.

17. Herding, J., Ludwig, S. \& Blankenburg, F. Response-Modality-Specific Encoding of Human Choices in Upper Beta Band Oscillations during Vibrotactile Comparisons. Frontiers in Human Neuroscience 11, (2017).

18. Siegel, M., Buschman, T. J. \& Miller, E. K. Cortical information flow during flexible sensorimotor decisions. Science 348, 1352-1355 (2015).

19. Allefeld, C. \& Haynes, J.-D. Searchlight-based multi-voxel pattern analysis of fMRI by cross-validated MANOVA. NeuroImage 89, 345-357 (2014). 
20. Christophel, T. B., Iamshchinina, P., Yan, C., Allefeld, C. \& Haynes, J.-D. Cortical specialization for attended versus unattended working memory. Nature Neuroscience 21, 494-496 (2018).

21. Britten, K. H., Newsome, W. T., Shadlen, M. N., Celebrini, S. \& Movshon, J. A. A relationship between behavioral choice and the visual responses of neurons in macaque MT. Visual Neuroscience 13, 87-100 (1996).

22. Nienborg, H. \& Cumming, B. G. Decision-related activity in sensory neurons reflects more than a neuron's causal effect. Nature 459, 89-92 (2009).

23. Cisek, P. \& Kalaska, J. F. Neural Correlates of Reaching Decisions in Dorsal Premotor Cortex: Specification of Multiple Direction Choices and Final Selection of Action. Neuron 45, 801-814 (2005).

24. King, J.-R. \& Dehaene, S. Characterizing the dynamics of mental representations: the temporal generalization method. Trends in Cognitive Sciences 18, 203-210 (2014).

25. Spaak, E., Watanabe, K., Funahashi, S. \& Stokes, M. G. Stable and Dynamic Coding for Working Memory in Primate Prefrontal Cortex. The Journal of Neuroscience 37, 65036516 (2017).

26. Zhao, Y., Yates, J. L., Levi, A. J., Huk, A. C. \& Park, I. M. Stimulus-choice (mis)alignment in primate area MT. PLOS Computational Biology 16, e1007614 (2020).

27. Lueckmann, J.-M., Macke, J. H. \& Nienborg, H. Can Serial Dependencies in Choices and Neural Activity Explain Choice Probabilities? The Journal of Neuroscience 38, 34953506 (2018).

28. Urai, A. E., de Gee, J. W., Tsetsos, K. \& Donner, T. H. Choice history biases subsequent evidence accumulation. eLife 8, (2019).

29. Macmillan, N. A. \& Creelman, C. D. Detection Theory: A User's Guide. (Psychology Press, 2004). 
30. Hebart, M. N., Schriever, Y., Donner, T. H. \& Haynes, J.-D. The Relationship between Perceptual Decision Variables and Confidence in the Human Brain. Cerebral Cortex 26, $118-130$ (2016).

31. Shadlen, M. N. \& Kiani, R. Decision Making as a Window on Cognition. Neuron 80, 791-806 (2013).

32. Kiani, R. \& Shadlen, M. N. Representation of Confidence Associated with a Decision by Neurons in the Parietal Cortex. Science 324, 759-764 (2009).

33. Donner, T. H., Siegel, M., Fries, P. \& Engel, A. K. Buildup of Choice-Predictive Activity in Human Motor Cortex during Perceptual Decision Making. Current Biology 19, 15811585 (2009).

34. Tsetsos, K., Pfeffer, T., Jentgens, P. \& Donner, T. H. Action Planning and the Timescale of Evidence Accumulation. PLOS ONE 10, e0129473 (2015).

35. O’Connell, R. G., Dockree, P. M. \& Kelly, S. P. A supramodal accumulation-to-bound signal that determines perceptual decisions in humans. Nature Neuroscience 15, 17291735 (2012).

36. Twomey, D. M., Kelly, S. P. \& O’Connell, R. G. Abstract and Effector-Selective Decision Signals Exhibit Qualitatively Distinct Dynamics before Delayed Perceptual Reports. Journal of Neuroscience 36, 7346-7352 (2016).

37. Sandhaeger, F., von Nicolai, C., Miller, E. K. \& Siegel, M. Monkey EEG links neuronal color and motion information across species and scales. eLife 8, e45645 (2019).

38. Kriegeskorte, N. et al. Matching Categorical Object Representations in Inferior Temporal Cortex of Man and Monkey. Neuron 60, 1126-1141 (2008).

39. Cichy, R. M., Pantazis, D. \& Oliva, A. Resolving human object recognition in space and time. Nature Neuroscience 17, 455-462 (2014). 
40. Wilming, N., Murphy, P. R., Meyniel, F. \& Donner, T. H. Large-scale Dynamics of Perceptual Decision Information across Human Cortex. bioRxiv (2020) doi:10.1101/2020.02.01.929893.

41. Li Hegner, Y., Lindner, A. \& Braun, C. A somatosensory-to-motor cascade of cortical areas engaged in perceptual decision making during tactile pattern discrimination: Cortical Cascade During Tactile Decisions. Human Brain Mapping 38, 1172-1181 (2017).

42. Klaes, C., Westendorff, S., Chakrabarti, S. \& Gail, A. Choosing Goals, Not Rules: Deciding among Rule-Based Action Plans. Neuron 70, 536-548 (2011).

43. Pape, A.-A. \& Siegel, M. Motor cortex activity predicts response alternation during sensorimotor decisions. Nature Communications 7, (2016).

44. Pape, A.-A., Noury, N. \& Siegel, M. Motor actions influence subsequent sensorimotor decisions. Scientific Reports 7, (2017).

45. Cisek, P. Making decisions through a distributed consensus. Current Opinion in Neurobiology 22, 927-936 (2012).

46. Summerfield, C. \& de Lange, F. P. Expectation in perceptual decision making: neural and computational mechanisms. Nature Reviews Neuroscience 15, 745-756 (2014).

47. Haefner, R. M., Berkes, P. \& Fiser, J. Perceptual Decision-Making as Probabilistic Inference by Neural Sampling. Neuron 90, 649-660 (2016).

48. Padoa-Schioppa, C. Neurobiology of Economic Choice: A Good-Based Model. Annual Review of Neuroscience 34, 333-359 (2011).

49. Freedman, D. J. \& Assad, J. A. A proposed common neural mechanism for categorization and perceptual decisions. Nature Neuroscience 14, 143-146 (2011).

50. Driel, J. van, Olivers, C. N. L. \& Fahrenfort, J. J. High-pass filtering artifacts in multivariate classification of neural time series data. bioRxiv (2019) doi:10.1101/530220. 
51. de Cheveigné, A. \& Arzounian, D. Robust detrending, rereferencing, outlier detection, and inpainting for multichannel data. NeuroImage 172, 903-912 (2018).

52. Nolte, G. The magnetic lead field theorem in the quasi-static approximation and its use for magnetoencephalography forward calculation in realistic volume conductors. Physics in Medicine and Biology 48, 3637-3652 (2003).

53. Van Veen, B. D., van Drongelen, W., Yuchtman, M. \& Suzuki, A. Localization of brain electrical activity via linearly constrained minimum variance spatial filtering. IEEE Transactions on biomedical engineering 44, (1997).

54. Hipp, J. F. \& Siegel, M. BOLD fMRI Correlation Reflects Frequency-Specific Neuronal Correlation. Current Biology 25, 1368-1374 (2015).

55. Hebart, M. N. \& Baker, C. I. Deconstructing multivariate decoding for the study of brain function. NeuroImage 180, 4-18 (2018).

56. Quax, S. C., Dijkstra, N., van Staveren, M. J., Bosch, S. E. \& van Gerven, M. A. J. Eye movements explain decodability during perception and cued attention in MEG.

NeuroImage 195, 444-453 (2019).

57. Oostenveld, R., Fries, P., Maris, E. \& Schoffelen, J.-M. FieldTrip: Open Source Software for Advanced Analysis of MEG, EEG, and Invasive Electrophysiological Data.

Computational Intelligence and Neuroscience 2011, 1-9 (2011). 


\section{Acknowledgments}

We thank Katrina Quinn for helpful discussions of the manuscript. This study was supported

by the European Research Council (ERC) StG335880 (M.S), Deutsche

Forschungsgemeinschaft (DFG; German Research Foundation) project 276693517 (SFB 1233)

(M.S.) and the Centre for Integrative Neuroscience (DFG, EXC 307) (M.S.). The authors acknowledge support by the state of Baden-Württemberg through bwHPC and the German Research Foundation (DFG) through grant no INST 39/963-1 FUGG (bwForCluster NEMO).

\section{Author contributions}

Conceptualization: M.S., F.S., A.A.P.; investigation: A.A.P.; formal analysis: F.S., N.O.; writing - original draft preparation: F.S.; writing - review and editing: F.S., N.O., A.A.P, M.S.; supervision: M.S.; resources: M.S.; funding acquisition: M.S.

\section{Competing interests}

A.A.P is a full-time employee of TWT GmbH. The remaining authors declare no competing interests.

\section{Materials \& Correspondence}

Correspondence to: florian.sandhaeger@uni-tuebingen.de, markus.siegel@uni-tuebingen.de 


\section{Supplementary Figures}

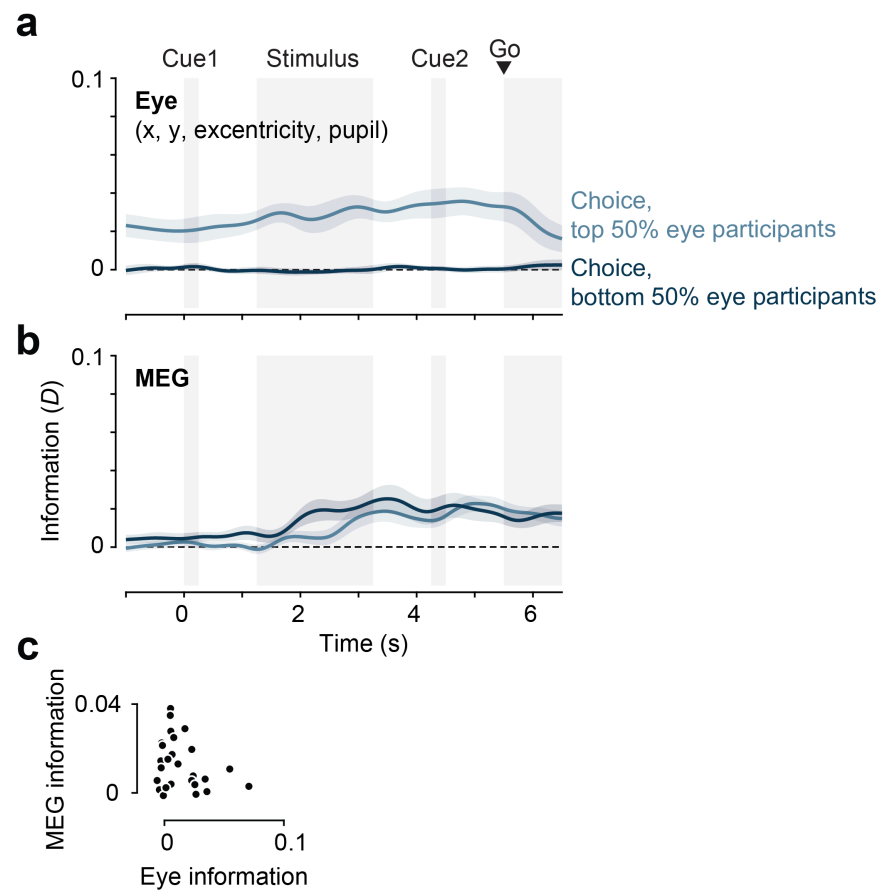

Supplementary Figure 1 | Choice information in eye-tracking data. a, Choice information contained in eye traces ( $\mathrm{x}$ and y position, eccentricity, and pupil size), split into the 13 participants with the highest decoding values, and the 13 participants with the lowest. Eye traces were thus predictive of choice in a subset of participants. $\mathbf{b}$, Choice information contained in MEG data, split into the same groups as in (a). Participants in whom the eye traces were predictive of choice did not show stronger decoding of choice from MEG data (one-tailed t-test, $P>$ 0.05 for all timepoints). This indicates that choice information in MEG was not driven by eye movements or pupil size. c, There was no positive across-subject relationship between the amount of choice information contained in eye traces and the amount of choice information contained in MEG data. 


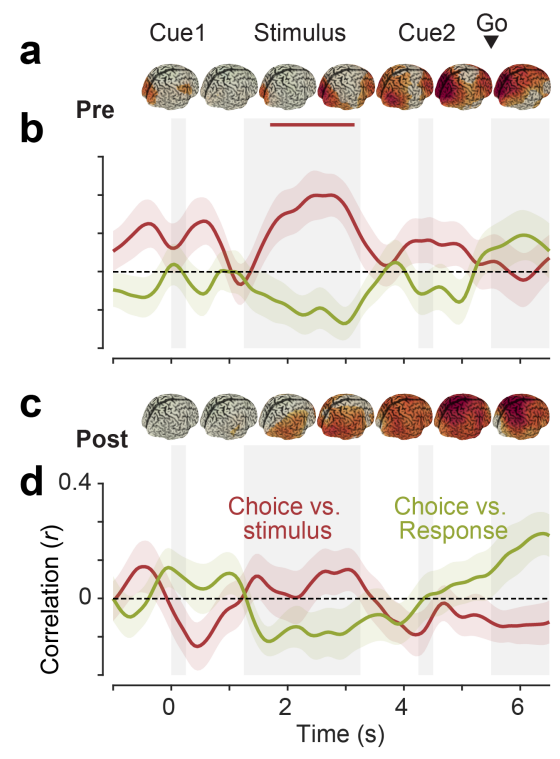

Supplementary Figure 2 | Spatiotemporal dynamics of choice information, split by task. a, cortical distribution of choice information during pre-cue trials. b. Correlation of the cortical distribution of choice information with the distribution of peak stimulus information (red) and peak response information (yellow) during pre-cue trials. $\mathbf{c}$ and $\mathbf{d}$, same as (a and b), during post-cue trials. Horizontal lines indicate clusters of significant correlation (cluster permutation, $P<0.05, \mathrm{~N}=26$ ).

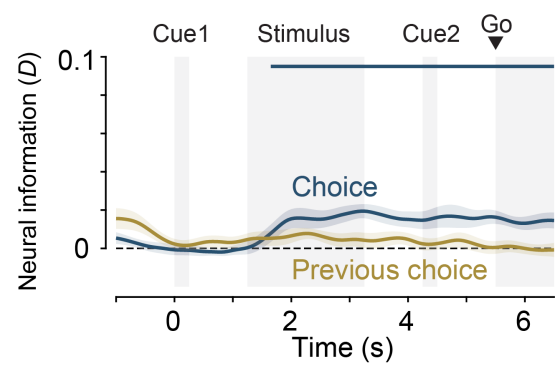

Supplementary Figure 3 | Previous choice information. There was no significant information about the previous choice, and information about the current choice remained significant when including previous choice as a variable. Horizontal lines indicate clusters of significant information (cluster permutation, $P<0.01, \mathrm{~N}=22$ ). 


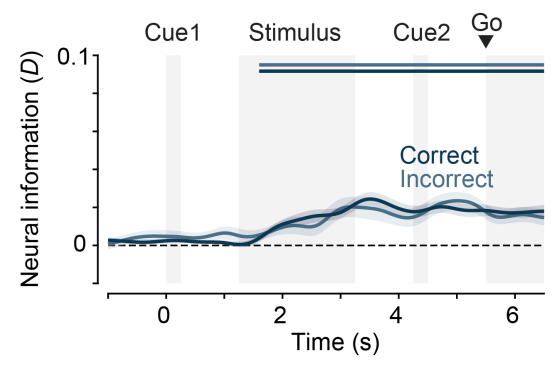

Supplementary Figure 4 | Choice information cannot be explained by accuracy. A model trained on all trials, but tested separately on correct and incorrect trials shows no difference between the two (two-tailed t-test, $P>$ 0.05 for all timepoints). Horizontal lines indicate clusters of significant information (cluster permutation, $P<$ $0.01, \mathrm{~N}=26$ ). Thus, choice information cannot be driven by accuracy.

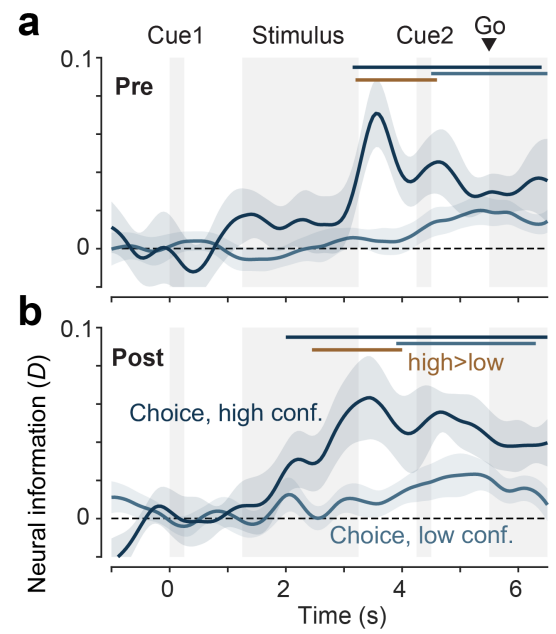

Supplementary Figure 5 | Confidence modulates the amount of choice information in both task conditions.

a, Time-resolved choice decoding in high (dark blue) and low confidence trials (light blue), respectively, during pre-cue trials. b, same as in (a), but during post-cue trials. Horizontal lines denote temporal clusters of significant information (colored) or differences in information magnitude (gold) $(P<0.05$, cluster-permutation, one-tailed, $\mathrm{N}=19$ ). 